\title{
Atomic Force Microscope Images Molecules in 3D
}

\author{
A new trick simplifies the atomic force microscope imaging of the 3D structure of nonflat \\ molecules.
}

\section{by Sébastien Gauthier*}

$\mathrm{N}$ early ten years ago, the dream of visualizing the atomic structure of single molecules became a reality. Spectacular textbook images of molecules adsorbed on surfaces could be produced by an atomic force microscope (AFM) with a specially prepared tip [1]. This technique has been applied with great success to many different molecules, allowing researchers to determine the strength of the bonds in molecular structures or monitor surface chemical reactions. In these experiments, the AFM tip stays at a constant height during an imaging scan, which is suitable for flat or nearly flat molecules but not for nonflat molecules with a 3D topography. A team led by Daniel Ebeling from Justus Liebig University Giessen in Germany now demonstrates that an AFM can image the 3D structure of nonflat molecules. In their approach, a tunneling current between the AFM tip and the sample is used to control the tip height, so that it closely tracks the molecule's topography. This new approach could extend AFM imaging to a much broader class of molecules [2].

Atomic force microscopy was invented in 1986 [3], a few

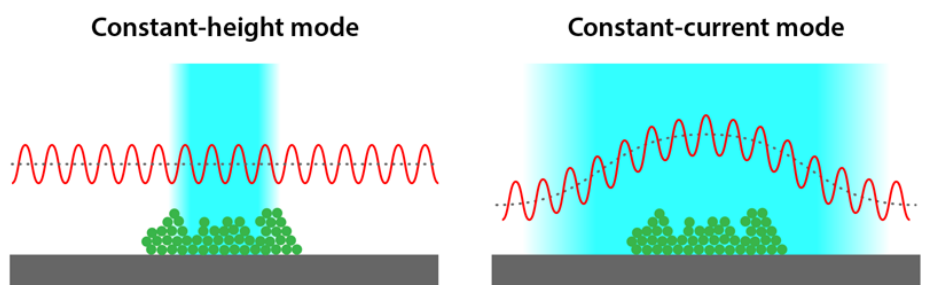

Figure 1: The AFM imaging of an adsorbed molecule on a substrate is usually done with the AFM tip oscillating at a constant height, where optimal imaging conditions (light blue region) are met only for the top part of the molecule. Daniel Ebeling's group uses a constant-current mode instead, in which the AFM tip closely tracks the molecule topography, allowing a complete 3D molecular imaging. (APS/Alan Stonebraker)

*Centre d'Élaboration de Matériaux et d'Etudes Structurales CNRS, Toulouse, France years after the scanning tunneling microscope (STM). These techniques belong to the scanning probe family, in which a sharp tip scans a sample's surface to produce an image. While an STM uses the tunneling current that flows through the vacuum separating the tip from the sample to form images, an AFM takes advantage of the force that the surface exerts on the tip. Both techniques can be used simultaneously, in a complementary way, with an STM providing information on the electronic structure of the sample and an AFM on its atomic structure.

With an AFM, the highest spatial resolution is obtained in the so-called noncontact mode, where the AFM tip does not actually touch the sample's surface. Instead, the tip is mounted on a quartz tuning fork and oscillates up and down just above the surface. Variations of the resonance frequency of this oscillator are then used to monitor the tip-surface force [4]. The key ingredient for high-resolution molecular imaging is to use an AFM with a single CO molecule at its tip [1]. It turns out that, in addition to producing a sharp, well-defined tip, this CO molecule acts as a tiny force sensor by bending under the influence of short-range chemical forces. Usually, the setup works in a constant-height mode: The tip is scanned in a plane parallel to the surface on which the molecule sits while recording the shift of the resonant frequency of the oscillating tuning fork. For tipmolecule distances of a few angstroms, short-range chemical forces that carry the molecular structural information become dominant, and one can obtain an image reflecting the bond structure of the molecule [1].

This technique has been applied to many different flat or nearly flat molecules, but it becomes limited when used on molecules with a 3D topography. The reason for that can be understood from Fig. 1 (left). When the frequency shift is monitored at constant height, the optimal imaging conditions are only met near the top of the $3 \mathrm{D}$ object. In the other areas, the tip is too far from the surface to collect a useful signal. Consequently, one obtains only a partial image of a nonflat molecule, missing important structural details at the molecule's periphery or in molecular parts that lie at different heights. This limitation greatly complicates the interpretation of the images of $3 \mathrm{D}$ objects. A natural solution to this problem would be to make the AFM tip closely follow the molecule's topography, as shown in Fig. 1 (right). 


\section{Phys̄īcs}

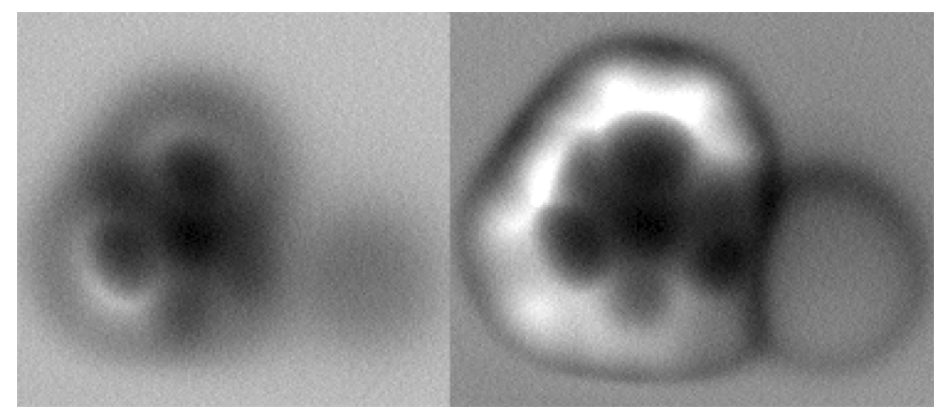

Figure 2: AFM images of a deiodinated ITP molecule in constant-height (left) and constant-current (right) modes. (D. Martin-Jimenez et al. [2])

Different strategies have been proposed to achieve this objective, but they invariably involve complex procedures and additional apparatuses [5].

In their work, the Giessen team overcomes these limitations and demonstrates a much simpler and more broadly applicable setup for the 3D imaging of nonflat molecules. Their method, based on a standard noncontact AFM setup, is one of the simplest that can be imagined: instead of operating the AFM in constant-height mode, they use the constant-tunneling-current mode of an STM. Since the tunneling current between the AFM tip and the surface depends on their separation, this ensures that the tip's height tracks the molecule's topography while scanning, allowing the acquisition of a useful signal everywhere on the molecule. The simplest ideas are often the best: the proposed technique can be implemented on any noncontact AFM setup, without requiring new instrumental development.

The authors first study a flat molecule, 2-iodotriphenylene (ITP) $\left(\mathrm{C}_{18} \mathrm{H}_{11} \mathrm{I}\right)$, deposited on a silver substrate, showing that the constant-height and constant-current methods give comparable results, although the images of the molecule's edges are blurrier in the constant-height case. Then, they remove one of the molecule's iodine atoms by applying, through the tip, a voltage pulse to a carbon-iodine bond. As a result, the molecule forms a radical-a chemically reactive molecule with an unpaired valence electron-with a complex 3D structure. They demonstrate that some parts of the radical are not visible at all in constant-height mode [Fig. 2 (left)] but become visible with the constant-current mode [Fig. 2 (right)]. In particular, they can reveal that a certain part of the molecule-a so-called deiodinated aryl ring-is strongly bent toward the surface.

Another major benefit of the new technique is that it would allow experimenters to scan larger sample areas without the risk of crashing the tip into an unexpected obstacle, such as an atomic step or a different type of molecule that could be present on the surface. Moreover, both the molecule and the substrate can be simultaneously imaged, which facilitates the determination of the orientation and the location of the molecule on the surface-important information to understand how the molecular properties are modified by the surface.

As with any novel idea, there are some drawbacks. One obvious limitation of the method is that it is restricted to electrically conducting substrates, to allow a current to flow from the tip through the sample and into the substrate. But many insulating materials can be used in the form of films that are thin enough to let a tunneling current pass through [6]. Another possible drawback appears when it comes to comparing the experimental results with numerical calculations: calculating a constant-current image requires many more numerical resources than a constant-height one. That's because in a constant-current calculation, one has to find, for each point of the image, the height that corresponds to the chosen current setpoint and carry out calculations based on that height.

The new constant-current mode of an AFM should have an immediate impact, for instance, in the field of on-surface chemistry, where researchers use a surface to synthesize new molecules from smaller ones [7]. It could also elucidate the structure of molecules that no other method can tackle [8]. As for its general usability, we will learn as we go from future use. In the present work, a certain current and bias voltage are used to control the AFM tip height in the range where the AFM frequency shift carries the molecular structural information. It is not yet clear if these experimental conditions will always be compatible with the stability of certain fragile molecules. But the simplified extension of atomic force microscopy to nonflat molecules demonstrated by the authors has the potential to be an important achievement in AFM imaging. More than three decades after its invention, atomic force microscopy has not ceased to surprise us!

This research is published in Physical Review Letters.

\section{REFERENCES}

[1] L. Gross, F. Mohn, N. Moll, P. Liljeroth, and G. Meyer, "The chemical structure of a molecule resolved by atomic force microscopy," Science 325, 1110 (2009).

[2] D. Martin-Jiminez, S. Ahles, D. Mollenhauer, H. A. Wegner, A. Schirmeisen, and D. Ebeling, "Bond-level imaging of the 3D conformation of adsorbed organic molecules using atomic force microscopy with simultaneous tunneling feedback," Phys. Rev. Lett. 122, 196101 (2019).

[3] G. Binnig, C. F. Quate, and Ch. Gerber, "Atomic force microscope," Phys. Rev. Lett. 56, 930 (1986).

[4] F. J. Giessibl, "Advances in atomic force microscopy," Rev. Mod. Phys. 75, 949 (2003).

[5] C. Moreno, O. Stetsovych, T. K. Shimizu, and O. Custance, "Imaging three-dimensional surface objects with submolecular resolution by atomic force microscopy," Nano Lett. 15, 2257 (2015). 
[6] J. Repp, G. Meyer, S. M. Stojković, A. Gourdon, and C. Joachim, "Molecules on insulating films: Scanning-tunneling microscopy imaging of individual molecular orbitals," Phys. Rev. Lett. 94, 026803 (2005).

[7] A. Gourdon, "On-surface covalent coupling in ultrahigh vacuum," Angew. Chem. Int. Ed. 47, 6950 (2008).
[8] M. Commodo, K. Kaiser, G. De Falco, P. Minutolo, F. Schulz, A. D, and L. Gross, "On the early stages of soot formation: Molecular structure elucidation by high-resolution atomic force microscopy," Combust. Flame 205, 154 (2019).

10.1103/Physics. 12.53 